\title{
Rendimiento y absorción de macro nutrientes en el cultivo de zanahoria dancus carota. Var nantes, y lombricomposto en un suelo Entisol
}

\author{
Yield and absorption of macro nutrients in carrot cultivation (dancus carota. Var \\ nantes), and (vermicompost) in an Entisol soil
Produção e absorção de macro nutrientes no cultivo de cenoura (dancus carota. Var nantes) e (vermicomposto) em solo Entiso

\author{
Kentaro Tomita ${ }^{1}$ \\ tomiken30@hotmail.com
}

Félix Arrúa Mancuello² arruamfelix@yahoo.com

\begin{abstract}
1Departamento de Desarrollo económica y Gestión ambiental, Gobierno Provincial de Imbabura, Ecuador. (Voluntario Senior de Agencia de Cooperación Internacional del Japón: JICA): (1Pre-Profesor Visitante de la Facultad de Ciencias Agropecuarias y Desarrollo Rural, Universidad Nacional de Pilar en Paraguay y Voluntario Senior de JICA). Japón
\end{abstract}

2Ing. Agr. : Facultad de Ciencias Agropecuarias y Desarrollo Rural, Universidad Nacional de Pilar, Paraguay

Artículo recibido noviembre 2017, arbitrado diciembre 2017 y publicado en mayo 2018

\section{RESUMEN}

El cultivo de la zanahoria es uno de los rubros que potencialmente, ofrece alternativas y ventajas para ser producidas en fincas de pequeños productores. En el Departamento de Ñeembucú, la producción de esta hortaliza no alcanza a satisfacer las demandas locales. El objetivo de este estudio es determinar el manejo de la fertilidad del suelo Entisol cultivado con esta hortaliza para establecer un sistema sostenible de producción con bajos insumos. Se evaluaron 4 niveles del $\mathrm{N}(0,50,100$ y $200 \mathrm{kgN} / \mathrm{ha})$ por 2 niveles del lombricomposto como M.O. ( 0 y 20t/ha) en el cultivo de zanahoria. La variedad utilizada fue Nantes. Al aplicar 20t/ha del lombricomposto (M.O.), se aplicaron $194 \mathrm{kgN} / \mathrm{ha}, 228 \mathrm{kgP} 205 / \mathrm{ha}, 50 \mathrm{kgK} 2 \mathrm{O} / \mathrm{ha}$, $29 \mathrm{kgCa} / \mathrm{ha}$ y $7.6 \mathrm{kgMg} / \mathrm{ha}$, respectivamente. Especialmente, se aplicó alto contenido del P205 en la superficie. No se observó diferencia estadísticamente significativa para materia orgánica del suelo en la mitad del ciclo del cultivo de zanahoria entre los tratamientos sin y con M.O. Los resultados de análisis de varianza, demuestra que hubo diferencia significativa al $1 \%$ para $\mathrm{N}$ y al $5 \%$ para M.O. en el rendimiento del tubérculo. En el tratamiento sin M.O., se observó alto rendimiento con $100 \mathrm{kgN} / \mathrm{ha}$, mientras que para el tratamiento con M.O. el de $50 \mathrm{kgN} / \mathrm{ha}$ arrojo el más alto rendimiento. Sobre análisis de tejido vegetal, los resultados de análisis de varianza, demuestran que hubo diferencia significativa al $1 \%$ no sólo para N sino también para M.O. en la absorción del $\mathrm{N}, \mathrm{P}$ y Mg en el tubérculo. Pero, se considera que hubo efecto diluido para la absorción del P en el tubérculo, teniendo en cuenta alto rendimiento observado en los tratamientos con M.O.

Palabras clave: cultivo de zanahoria; fertilidad del suelo, dancus carota var nantes, y lombricomposto

\begin{abstract}
Carrot cultivation is one of the items that potentially offers alternatives and advantages to be produced on farms of small producers. In the Ñeembucú Department, the production of this vegetable does not meet local demands. The objective of this study is to determine the fertility management of the Entisol soil cultivated with this vegetable to establish a sustainable production system with low inputs. Four levels of $\mathrm{N}(0,50,100$ and $200 \mathrm{kgN} / \mathrm{ha})$ were evaluated for two levels of the vermicompost as M.O. ( 0 and $20 \mathrm{t} / \mathrm{ha}$ ) in carrot cultivation. The variety used was Nantes. When applying $20 \mathrm{t} /$ ha of the earthworm (M.O.), 194kgN / ha, 228kgP2O5 / ha, $50 \mathrm{kgK} 20$ / ha, $29 \mathrm{kgCa} / \mathrm{ha}$ and $7.6 \mathrm{kgMg} / \mathrm{ha}$, respectively, were applied. Especially, high content of P2O5 was applied on the surface. No statistically significant difference was observed for soil organic matter in the middle of the carrot crop cycle between treatments without and with M.O. The results of analysis of variance show that there was a significant difference of $1 \%$ for $\mathrm{N}$ and $5 \%$ for M.O. in tuber yield. In the treatment without M.O., high yield was observed with $100 \mathrm{kgN} / \mathrm{ha}$, while for the treatment with M.O. that of $50 \mathrm{kgN} /$ ha yielded the highest yield. On analysis of plant tissue, the results of analysis of variance show that there was a significant difference of $1 \%$ not only for $\mathrm{N}$ but also for M.O. in the absorption of $\mathrm{N}, \mathrm{P}$ and $\mathrm{Mg}$ in the tuber. But, it is considered that there was a diluted effect for the absorption of $\mathrm{P}$ in the tuber, taking into account the high performance observed in the treatments with M.O.
\end{abstract}

Key words: carrot cultivation; soil fertility, dancus carota var nantes, and vermicompost

\section{Alfa, Revista de Investigación en Ciencias Agronómicas y Veterinarias Vol. 2, NNro. 5, Mayo - Agosto 2018}




\section{RESUMO}

O cultivo de cenoura é um dos itens que potencialmente oferece alternativas e vantagens a serem produzidas em fazendas de pequenos produtores. No departamento de Ñeembucú, a produção desse vegetal não atende às demandas locais. 0 objetivo deste estudo é determinar o manejo da fertilidade do solo do Entisol cultivado com esse vegetal para estabelecer um sistema de produção sustentável com baixos insumos. Quatro níveis de N (0,50, 100 e $200 \mathrm{kgN} / \mathrm{ha})$ foram avaliados para dois níveis de vermicomposto como M.O. (0 e 20t / ha) no cultivo de cenoura. A variedade utilizada foi Nantes. Ao aplicar $20 \mathrm{t} / \mathrm{ha}$ do vermicomposto (M.O.), foram aplicados $194 \mathrm{kgN} / \mathrm{ha}$, $228 \mathrm{kgP} 205$ / ha, $50 \mathrm{kgK} 20$ / ha, $29 \mathrm{kgCa} /$ ha e $7,6 \mathrm{kgMg}$ / ha, respectivamente. Especialmente, alto teor de P205 foi aplicado na superfície. Não foi observada diferença estatisticamente significante para a matéria orgânica do solo no meio do ciclo da cultura da cenoura entre os tratamentos sem e com M.O. Os resultados da análise de variância mostram que houve uma diferença significativa de $1 \%$ para $\mathrm{N}$ e $5 \%$ para M.O. no rendimento de tubérculos. No tratamento sem M.O., observou-se alto rendimento com $100 \mathrm{kgN} / \mathrm{ha}$, enquanto no tratamento com M.O. o de $50 \mathrm{kgN} /$ ha produziu o maior rendimento. $\mathrm{Na}$ análise do tecido vegetal, os resultados da análise de variância mostram que houve uma diferença significativa de $1 \%$ não apenas para N, mas também para M.O. na absorção de N, P e Mg no tubérculo. Porém, considera-se que houve um efeito diluído na absorção de $\mathrm{P}$ no tubérculo, levando em consideração o alto desempenho observado nos tratamentos com M.O.

Palavras-chave: cultivo de cenoura; fertilidade do solo, dancus carota var nantes e vermicomposto

\section{INTRODUCCIÓN}

La zanahoria es una planta bianual, durante el primer año se forma una roseta de pocas hojas y la raíz. Después de un período de descanso, se presenta un tallo corto en el que se forman las flores durante la segunda estación de crecimiento, presenta un sistema radicular napiforme, de forma $y$ color variables.

El cultivo de la zanahoria es uno de los rubros que potencialmente, ofrece alternativas y ventajas para ser producidas en fincas de pequeños productores. Por las características alimenticias del rubro, el mismo es muy apreciado; principalmente en los países de clima templado tienen un alto contenido en vitaminas.

Atendiendo a los aspecto mencionados, en el Departamento de Ñeembucú, la producción de esta hortaliza no alcanza a satisfacer las demandas locales (Cantidades de fincas fue de 16 y 1 ha para superficie sembrada, Censo del 2008), esto es verificable en el hecho que el volumen importante del mismo arriba de semana a semana en la Ciudad de Pilar provenientes de otros Departamentos y está distribuida en las despensas y negocios para su comercialización al público.

Por eso, es necesario investigar manejo de la fertilidad del suelo Entisol cultivado con esta hortaliza para establecer un sistema sostenible de producción con bajos insumos.

\section{MATERIALES Y MÉTODO}

A continuación se presenta una serie de pasos que dieron lugar al levantamiento del estudio.

\section{Clasificación del suelo en la finca experimental}

La finca experimental del suelo en la Facultad de Ciencias Agropecuarias y Desarrollo Rural se clasifica como Entisol (Típico, Psammaquent) por taxonomía del suelo en Estados Unidos de América.

\section{Densidad de la siembra de zanahoria}

Unidades experimentales de $2.40 \mathrm{~m}$ de largo por $1.0 \mathrm{~m}$ de ancho $=2.40 \mathrm{~m} 2$ (parcela principal); $\mathrm{y}$ las parcelas (divididas) $\mathrm{o}$ subparcelas de $1.20 \mathrm{~m} 2$ (=1.20m x $1.0 \mathrm{~m})$. Densidad total del experimento $12.1 \mathrm{~m} \mathrm{x}$ $6.5 \mathrm{~m}=78.65 \mathrm{~m} 2$. Densidad de siembra de $20 \mathrm{~cm}$ entre hileras, y $5 \mathrm{~cm}$ entre plantas, con área cultivada de $38.4 \mathrm{~m} 2 \quad(=2.4 \mathrm{~m} 2 \times 16$ : Unidad experimental). La siembra se realizó en banda. (La cantidad de semilla utilizada fue de 15 a $20 \mathrm{~g}$ por $38.4 \mathrm{~m} 2)$. El área útil fue de $0.20 \mathrm{~m} 2(0.4 \mathrm{~m} \times 0.5 \mathrm{~m})$. 


\section{Evaluación de diferentes niveles de fertilizantes y época de aplicación del $\mathrm{N}$ químico}

Se evaluaron 4 niveles de Nitrógeno con $0,50,100$ y $200 \mathrm{kgN} / \mathrm{ha}$, utilizando la Urea como fuente de nitrógeno (parcela principal), y 2 niveles de materia orgánica con 0 y 20t/ha de Lombricomposto (parcela dividida), distribuidos en un diseño completamente al azar con 4 réplicas.

El día 17 de mayo de 2011, se realizó toma de muestra del suelo en el campo, uniformemente para saber la característica física-química de esto. El día siguiente, se realizó la aplicación de la materia orgánica, y el día de 31 de mayo se sacó muestra del suelo entre los tratamientos divididas sin y con materia orgánica aplicada para comparar el contenido en el suelo, posterior a eso se realizó la siembra directa (Variedad: Nantes) en banda y la primera aplicación del fertilizante nitrogenado al boleo luego de la siembra. Además, se realizaron segunda y tercera fertilización nitrogenada química a los 30 y 60 días después de la siembra. (Álvaro, 1993)

\section{Cosecha de Zanahoria}

Se realizó cosecha de los tubérculos dentro del área útil en cada tratamiento el día 28 de septiembre de 2011, y se lo calculó por hectárea.

Además, sobre los tubérculos escogidos, se metieron el horno para secar y calcular porcentaje de materia seca y humedad respectivamente. Finalmente, se utilizó el cálculo de absorción de los macros nutrientes en el tubérculo ( $\mathrm{kg} / \mathrm{ha}$ ).

\section{Análisis de la característica física- química del suelo}

Para analizar las características físicasquímicas del suelo, se tomaron muestras antes de la siembra, dentro (mitad del ciclo del cultivo) y después del cultivo para saber la dinámica de nutrientes en la superficie del suelo en cada tratamiento, el análisis de suelo se realizó en el Laboratorio de Suelos de la Facultad de Ciencia Básica y Aplicada de la Univ. Nacional de Pilar. Para el análisis en el Laboratorio de Suelos, se utilizó agua destilada (agua: suelo $=1: 1$ ) para determinar el valor de $\mathrm{pH}$, la solución sal de $\mathrm{KCl}$ al $1 \mathrm{M}$ para determinar $\mathrm{Al}$ intercambiable $\mathrm{y}$ el método de Walkley-Black para determinar materia orgánica. (Arias, 2007).

Por otra parte, sobre análisis de otros elementos, se realizó en la Fundación NikkeiCETAPAR (CENTRO TECNOLÓGICO AGROPECUARIO DEL PARAGUAY), y se utilizaron la solución extractora de Mehlich No1 $(0.05 \mathrm{M} \mathrm{HCl}+0.0125 \mathrm{M}$ H2SO4) para determinar $\mathrm{P}$ y $\mathrm{K}$ disponibles y la solución sal de $\mathrm{KCl}$ al $1 \mathrm{M}$ para determinar $\mathrm{Ca}$ y $\mathrm{Mg}$ intercambiables antes de la siembra y la aplicación del abono químico y orgánico Por otra parte, para determinar cantidad de arcilla, se utilizó el método de Bouyoucos.

\section{Análisis del lombricomposto y de tejido vegetal}

En el CETAPAR se utilizó el método de Kjeldahl para determinar el contenido de $\mathrm{N}$ Total, y el método de ácido nitrato más ácido perclorúrico para determinar el contenido de elementos mayores tales como $\mathrm{P}, \mathrm{K}, \mathrm{Ca}$ y $\mathrm{Mg}$ sobre el análisis de tejido vegetal. Sobre cálculo de la absorción de los macros nutrientes, el valor seco promedio en los tubérculos escogidos fue de 11.2 (\%). Se lo utilizó como el valor fresco x $0.112=$ el seco en el experimento, y se calculó la absorción de los macros nutrientes en el tubérculo seco (kg/ha) de acuerdo a los resultados analizados del CETAPAR. Además, se evaluó la recuperación eficiente del $\mathrm{N}$ aplicado en cada nivel del $\mathrm{N}$ aplicado entre los tratamiento sin y con aplicación de materia orgánica sobre la absorción nitrogenada en el tubérculo. 
RESULTADOS Y DISCUSIÓN

\section{Característica química del suelo antes de la aplicación del lombricomposto}

La Tabla 1 muestra la característica química del suelo antes de la aplicación del lombricomposto. De los resultados analizados, el valor de pH fue de 6.06 (poco ácido), el $\mathrm{P}$ y $\mathrm{K}$ disponibles por la solución extractora de Mehlich No1 fueron de $154.9 \mathrm{y}$ $0.435 \mathrm{cmolc} / \mathrm{kg}$, respectivamente. Además se observó alto contenido del Ca intercambiable en la superficie del suelo. Por eso, por algún tiempo, se considera que no fue necesario aplicar abono fosfatado ni potásico.

Tabla 1. Característica química del suelo antes de la aplicación del lombricomposto

Antes de la aplicación del lombricomposto

\begin{tabular}{|c|c|c|c|c|c|c|}
\hline Arcilla & $\mathrm{pH}$ & $\mathrm{P}$ & $\mathrm{K}$ & $\mathrm{Ca}$ & $\mathrm{Mg}$ & $\mathrm{Al}$ \\
\hline$(\%)$ & $\left(\mathrm{H}_{2} \mathrm{O}\right)$ & $(\mathrm{mg} / \mathrm{kg})$ & \multicolumn{4}{|c|}{$\left(\mathrm{cmol}_{\mathrm{C}} / \mathrm{kg}\right)$} \\
\hline 4,24 & 6,06 & 154,9 & 0,435 & 4,81 & 1,48 & 0,19 \\
\hline
\end{tabular}

De todos modos, se considera que hubo alto contenido de material arcilloso tipo 2:1 parte de arcilla aunque alto contenido de arena en el suelo teniendo en cuenta mucha aplicación anterior de abono químico y orgánico.

\section{Análisis químico del lombricomposto y cantidad aplicada por hectárea}

Tabla 2. Análisis químico del lombricomposto (Materia orgánica) y cantidad de nutrientes aplicados al suelo con 20t/ha de M.O.

\begin{tabular}{ccccc|cccc}
\hline $\mathrm{N}$ & $\mathrm{P}$ & $\begin{array}{c}\mathrm{K} \\
(\%)\end{array}$ & $\mathrm{Ca}$ & $\mathrm{Mg}$ & $\mathrm{Mn}$ & $\begin{array}{c}\mathrm{Fe} \\
(\mathrm{mg} / \mathrm{kg})\end{array}$ & $\mathrm{Zn}$ & $\mathrm{Cu}$ \\
\hline 0,97 & 0,497 & 0,21 & 0,144 & 0,038 & 68,67 & 998,45 & 47,82 & 7,12 \\
\hline \multicolumn{2}{l}{ Cantidad aplicada por } & $20 \mathrm{t} / \mathrm{ha}$ & & & & & & \\
\hline $\mathrm{N}$ & $\mathrm{P}$ & $\begin{array}{c}\mathrm{K} \\
(\mathrm{kg} / \mathrm{ha})\end{array}$ & $\mathrm{Ca}$ & $\mathrm{Mg}$ & $\mathrm{Mn}$ & $\begin{array}{c}\mathrm{Fe} \\
(\mathrm{g} / \mathrm{ha})\end{array}$ & $\mathrm{Zn}$ & $\mathrm{Cu}$ \\
\hline 194 & 99,4 & 42 & 28,8 & 7,6 & 1373,4 & 19969 & 956,4 & 142,4 \\
\hline
\end{tabular}

PS: Como $\mathrm{P}_{2} \mathrm{O}_{5}$ y $\mathrm{K}_{2} \mathrm{O}(\mathrm{kg} / \mathrm{ha})$, se aplicaron 228 y $50 \mathrm{~kg} / \mathrm{ha}$, respectivamente.

La Tabla 2 muestra el análisis químico y la cantidad de nutrientes aplicado al suelo con 20t/ha de lombricomposto utilizado como abono orgánico Sobre el valor de porcentaje, se observó $0.97 \%$ del N, $0.497 \%$ para el P, $0,21 \%$ de K, 0,144\% de Ca y 0,038\% de Mg. Al aplicar $20 \mathrm{t} / \mathrm{ha}$ de este material, se aplicaron $194 \mathrm{kgN} / \mathrm{ha}, 228 \mathrm{kgP} 205 / \mathrm{ha}, 50 \mathrm{kgK} 20 / \mathrm{ha}, 29 \mathrm{kgCa} / \mathrm{ha} \mathrm{y}$ $7.6 \mathrm{kgMg} / \mathrm{ha}$, respectivamente. Especialmente, se aplicó alto contenido del P205 en la superficie. 


\section{Característica agronómica para Zanahoria \\ Fotografía mostradas en el bloque para el cultivo de zanahoria}

Las Fotografías 1 y 2 muestran el cultivo del zanahoria en el suelo Entsol en el campo de la Facultad de Ciencias Agropecuarias y Desarrollo Rural, Universidad nacional de Pilar. En realdad, el suelo fue muy compactado, tomando en cuenta alto contenido de arena, por lo que hubo mala influencia para el tubérculo (ver Fotografía 2).

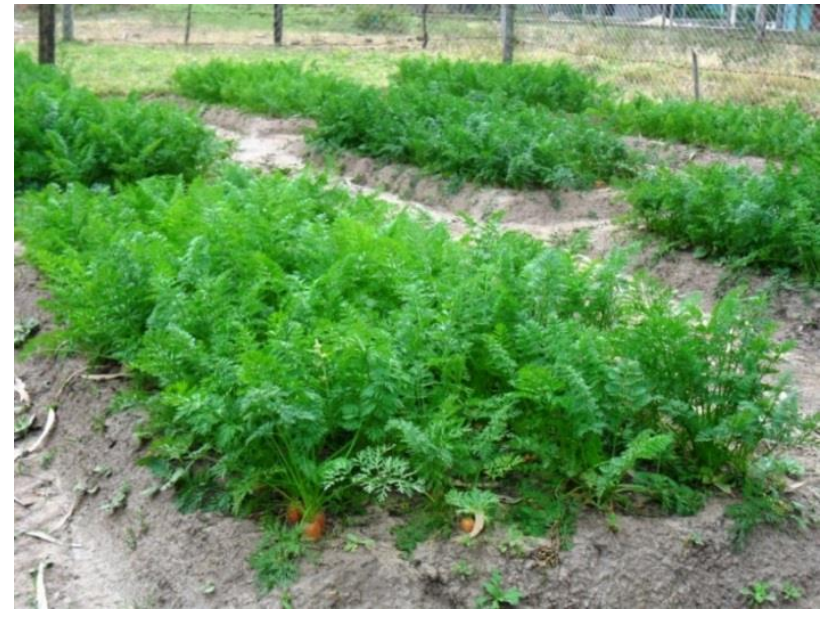

Fotografía 1. Diferentes niveles del N y lombricomposto en el cultivo de Zanahoria, 2011.

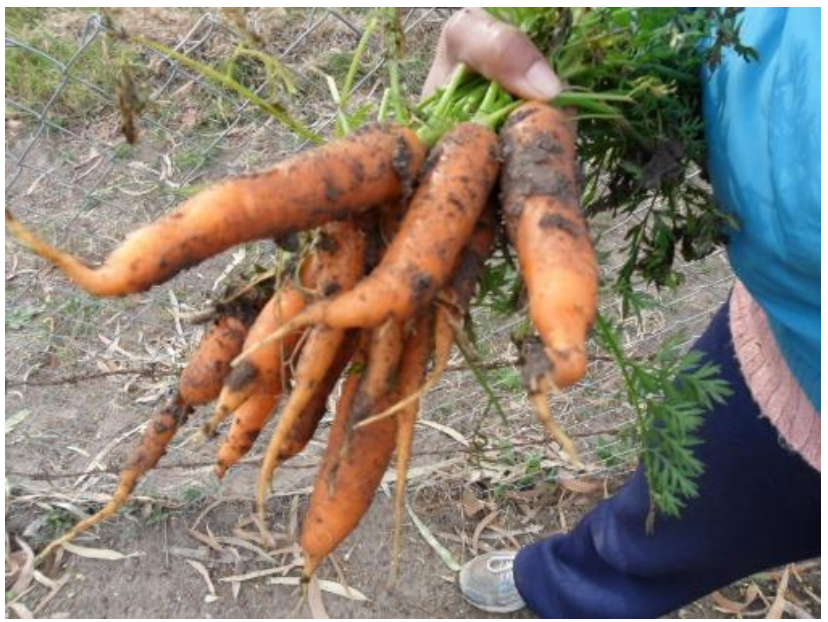

Fotografía 2. Cosecha del tubérculo de Zanahoria, 2011.

Rendimiento del tubérculo en los fincas de pequeños productores. Se evaluaron diferentes niveles del $\boldsymbol{N} \quad \boldsymbol{y} \quad 4$ niveles del N (0,50,100 y $200 \mathrm{kgN} / \mathrm{ha})$ por 2 lombricomposto niveles del lombricomposto como Materia orgánica (M.O.) (0 y 20t/ha) en el cultivo de

El cultivo de la zanahoria es uno de los rubros que potencialmente, ofrece zanahoria (Dancus carota). La variedad alternativas y ventajas para ser producidas en utilizada fue Nantes. 
En el lombricomposto se contienen $194 \mathrm{kgN} / \mathrm{ha}, \quad 99.4 \mathrm{kgP} / \mathrm{ha}, \quad 42 \mathrm{kgK} / \mathrm{ha}$, $29 \mathrm{kgCa} /$ ha y $7,6 \mathrm{kgMg} / \mathrm{ha}$, respectivamente. La Figura No1 muestra la dinámica del rendimiento del tubérculo en cada tratamiento nitrogenado entre los tratamientos sin y con M.O. Los resultados de análisis de varianza, demuestra que hubo diferencia significativa al $1 \%$ para $\mathrm{N}$ y al $5 \%$ para M.O. en el rendimiento del tubérculo.

En el tratamiento sin M.O., se observó alto rendimiento con $100 \mathrm{kgN} / \mathrm{ha}$, mientras que para el tratamiento con M.O. el de $50 \mathrm{kgN} / \mathrm{ha}$ arrojo el más alto rendimiento.

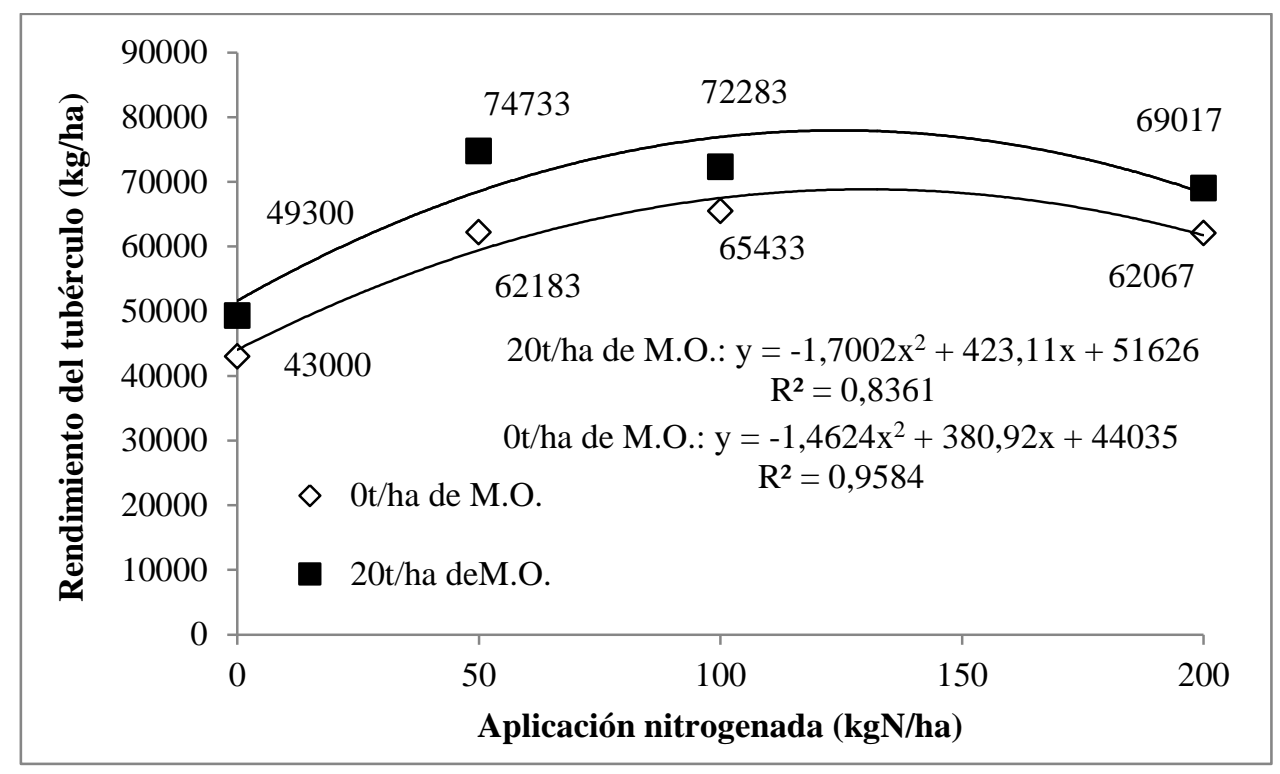

Figura 1. Dinámica del rendimiento del tubérculo en cada tratamiento nitrogenado entre los tratamientos sin y con M.O. 


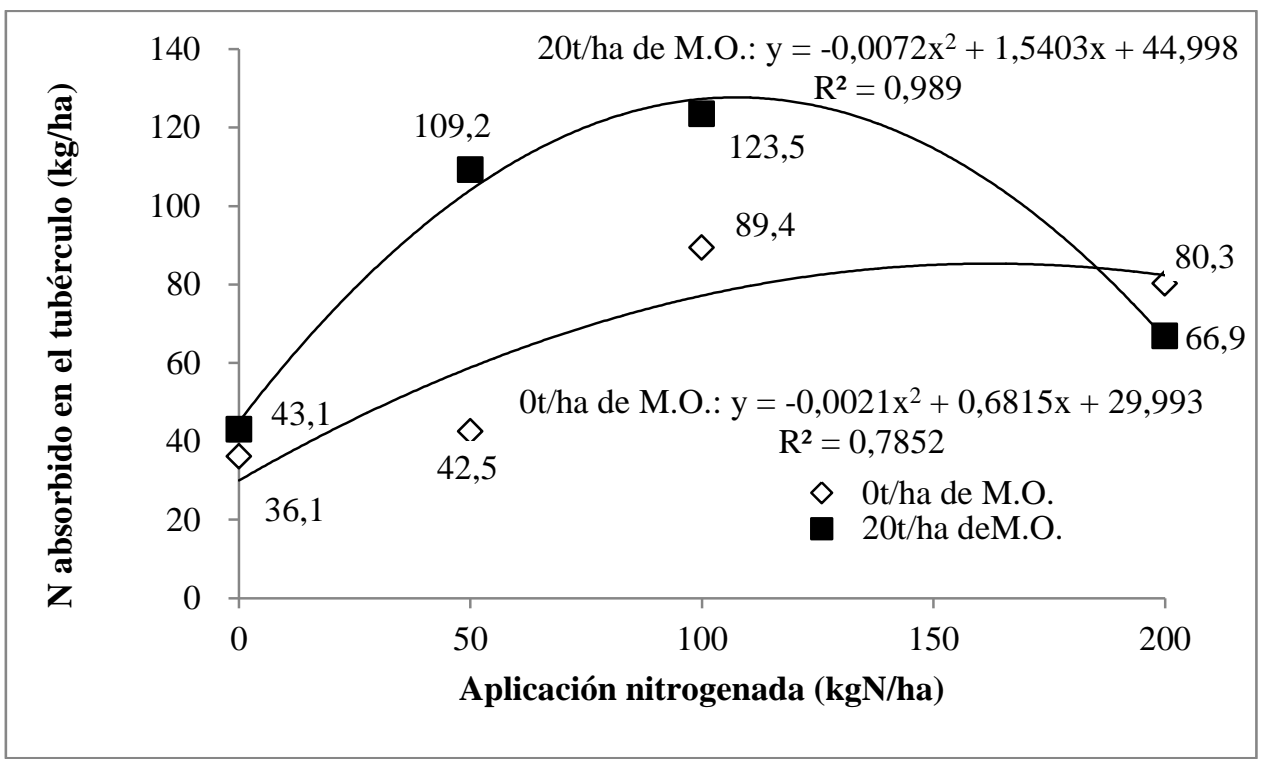

Figura 2. Comparación de la absorción del $\mathrm{N}$ en el tubérculo $(\mathrm{kg} / \mathrm{ha})$ en cada tratamiento nitrogenado entre los tratamientos sin y con M.O.

Absorción de los 5 macro nutrientes en el tubérculo en los diferentes niveles del N y lombricomposto

Sobre análisis de tejido vegetal, los resultados de análisis de varianza, demuestran que hubo diferencia significativa al 1\% no sólo para N sino también para M.O. en la absorción del $\mathrm{N}$ en el tubérculo (ver la Figura 2).

Fue mejor para la aplicación de $50 \mathrm{kgN} / \mathrm{ha}$ más 20t/ha del lombricomposto de punta de vista del más alto rendimiento.

La Figura 3 muestra comparación de la absorción del $\mathrm{P}$ en el tubérculo de acuerdo a la aplicación nitrogenada. De los resultados de análisis de varianza se observó una diferencia significativa al $1 \%$ no sólo para $\mathrm{N}$ sino también M.O. al igual que la absorción del $\mathrm{N}$. A diferencia del $\mathrm{N}$, se observó alto contenido de la absorción en los tratamientos sin M.O. más que la absorción en los con M.O., relativamente. Se considera que un efecto diluido para tubérculo en los tratamientos con M.O., teniendo en cuenta alto rendimiento observado.

La Figura 3 muestra dinámica de la absorción del $\mathrm{K}$ en el tubérculo de acuerdo a la aplicación nitrogenada. En cuanto a la absorción del $\mathrm{K}$ en el tubérculo se observó la diferencia significativa al $1 \%$ para $\mathrm{N}$, solamente. De acuerdo con la aplicación nitrogenada, se observó alta absorción del K en el tubérculo. 

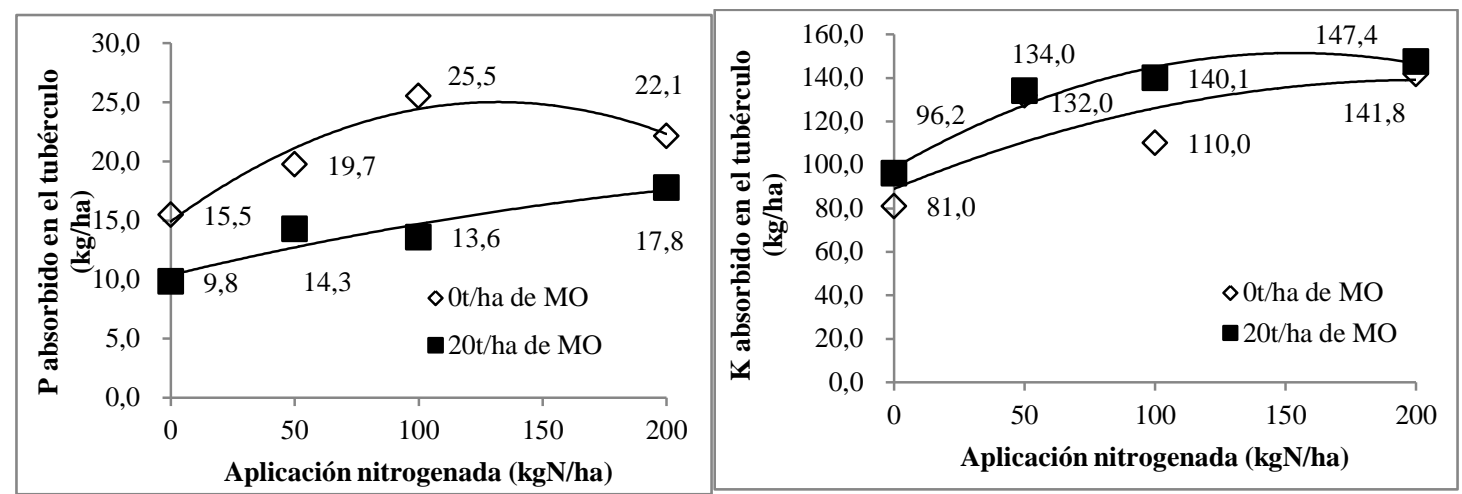

Figura 3. Dinámica de la absorción del P y del K en el tubérculo (kg/ha) en cada tratamiento nitrogenado entre los tratamientos sin y con M.O.
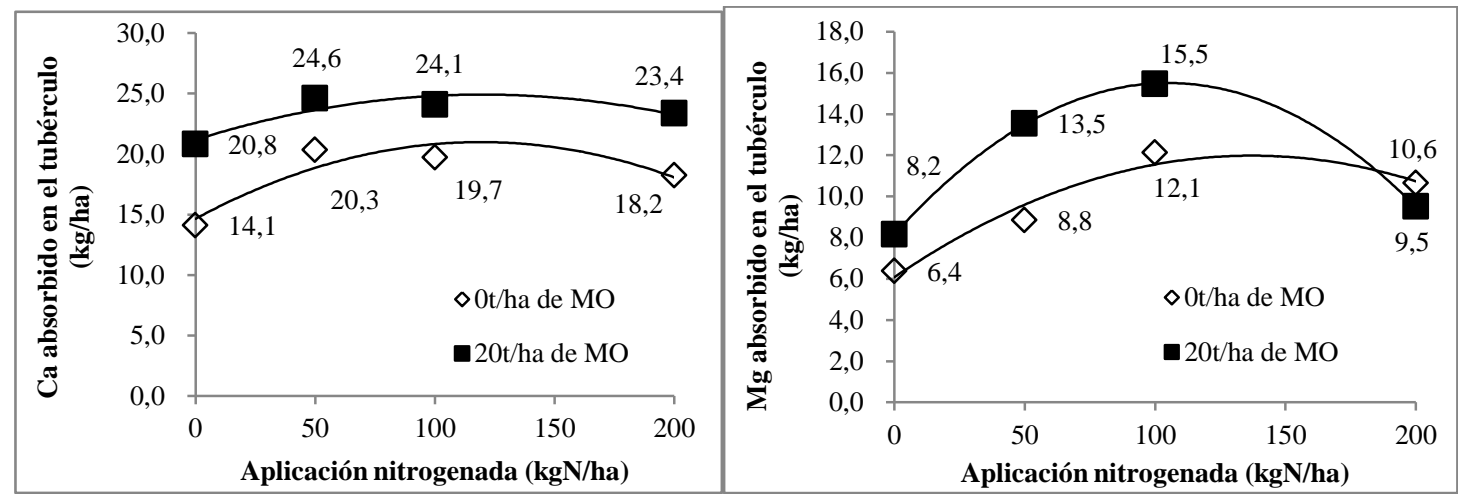

Figura 4. Dinámica de la absorción del Ca y del Mg en el tubérculo (kg/ha) en cada tratamiento nitrogenado entre los tratamientos sin y con M.O.

La Figura 4 muestra comparación de la absorción del Ca de acuerdo con la aplicación nitrogenada. En cuanto a la absorción del Ca en el tubérculo se observó la diferencia significativa al $5 \%$ para $\mathrm{N}$ y al $1 \%$ para M.O. Relativamente, se observó alta absorción del Ca en los tratamientos con M.O. más que la absorción en los sin M.O. Se considera que hubo efecto del Ca liberado o aplicado por la materia orgánica (el lombiricomposto), teniendo en cuenta alto valor del $\mathrm{pH}$, alto rendimiento, entre otros.

La Figura 4 muestra comparación de la absorción del Mg en el tubérculo de acuerdo a la aplicación nitrogenada. De los resultados de análisis de varianza, se observó la diferencia significativa al 1\% no sólo para N sino también para M.O. al igual que la absorción del $\mathrm{N}$ y $\mathrm{P}$. Al igual que el $\mathrm{Ca}$, se considera que hubo efecto del Mg liberado o aplicado por la materia orgánica en los tratamientos con M.O.

\section{Evaluación económica para el cultivo de Zanahoria}

La Tabla 3 muestra Costo fijo, y la Tabla No4 muestra la evaluación económica para el cultivo de zanahoria var Nantes. Sobre el costo de lombricomposto, fue de Gs 2.728 .000 para Lombricomposto 0t/ha $+0 \mathrm{kgN} / \mathrm{ha}$, mientras que para Lombricomposto 20t/ha + $0 \mathrm{kgN} / \mathrm{ha}$ Gs 3.988.000. Por fin para el consto 
de lombricomposto,

3.988.000-

$2.728 .000=1.260 .000$.

En el beneficio neto, a pesar de la aplicación con 20t/ha de lombricomposto, fue de Gs 306.411.705 en el tratamiento de $20 \mathrm{t} / \mathrm{ha}$ de lombricomposto $+50 \mathrm{kgN} / \mathrm{ha}$ a contra Gs 255.488 .805 en el tratamiento de 0t/ha de lombricomposto $+50 \mathrm{kgN} /$ ha. Por fin se observó la respuesta positiva para el rendimiento de tubérculo por la aplicación de lombricomposto, fue más alto para el beneficio.

Tabla 3. Costo fijo para el cultivo de zanahoria var Nantes.

\begin{tabular}{|c|c|c|}
\hline \multicolumn{3}{|l|}{ Costo fijo } \\
\hline \multicolumn{3}{|l|}{ Mano de obra } \\
\hline Descripción & Cantidad & Precio (US\$)_osto Total (USS \\
\hline $\begin{array}{l}\text { Raleo } \\
\text { Siembra } \\
\text { Carrida } \\
\text { Riego y cosecha } \\
\end{array}$ & 23 & 256 \\
\hline & & Sub Total \\
\hline \multicolumn{3}{|c|}{ Preparación del terreno } \\
\hline \multicolumn{2}{|l|}{ Maquina Agrícola } & Precio (US\$) _osto Total (USS \\
\hline Subsuelo & 1 & 67 \\
\hline Labranza & 1 & 67 \\
\hline Rastreada & 1 & 44 \\
\hline \multicolumn{3}{|r|}{ Sub Total } \\
\hline \multicolumn{3}{|l|}{ Insumos } \\
\hline Descripción & Cantidad (g/ha) & Precio (US\$)_osto Total (USS \\
\hline Semilla & 1950 & 0,054 \\
\hline Descripción & Cantidad (L/ha) & Precio (US\$)_osto Total (US\$ \\
\hline Glifosato & 5 & 13,3 \\
\hline \multicolumn{3}{|r|}{ Sub Total } \\
\hline Costo fijo & & 606 \\
\hline
\end{tabular}

Pero, al aplicar 20t/ha de lombricomposto, fue de Gs 295.884.497 en el tratamiento con $100 \mathrm{kgN} / \mathrm{ha}$, fue mejor para el tratamiento con $50 \mathrm{kgN} / \mathrm{ha}$ (En el tratamiento sin lombricomposto, fue el más alto para el tratamiento con $100 \mathrm{kgN} / \mathrm{ha}$. Pero, fue mejor para aplicar el lombricomposto, tomando en cuanta agregar otros nutrientes que provienen del lombricomposto, totalmente). 
Tabla 4. Evaluación de la aplicación óptima y económica de los materiales aplicados en el cultivo de zanahoria.

\begin{tabular}{|c|c|c|c|c|c|c|c|c|}
\hline $\begin{array}{c}\text { Lombricomposto } \\
\text { (t/ha) } \\
\end{array}$ & $\begin{array}{c}\text { Aplicación N } \\
\text { (Urea) } \\
\text { (kgN/ha) }\end{array}$ & $\begin{array}{c}\text { Rendimiento } \\
\text { de tubérculo } \\
\text { (kg/ha) }\end{array}$ & \begin{tabular}{|c|}
$10 \%$ Perdidad en \\
la cosecha \\
$(\mathrm{kg} / \mathrm{ha})$ \\
\end{tabular} & $\begin{array}{c}\text { Beneficio } \\
\text { bruto }\end{array}$ & Costo fijo & \begin{tabular}{|l|} 
Costo de Urea \\
Lombricompostd \\
\begin{tabular}{|c|c|} 
(US\$) \\
\end{tabular} \\
\end{tabular} & \begin{tabular}{|c|} 
Costo fijo + Ure \\
Lombricompost \\
(US\$/ha)
\end{tabular} & $\begin{array}{c}\text { Beneficio } \\
\text { neto } \\
\text { (US\$/ha) }\end{array}$ \\
\hline \multirow[t]{4}{*}{0} & 0 & 43000 & 38700 & 42570 & 606 & 0 & 606 & 41964 \\
\hline & 50 & 62183 & 55965 & 61561 & 606 & 81 & 687 & 60874 \\
\hline & 100 & 65433 & 58890 & 64779 & 606 & 162 & 768 & 64011 \\
\hline & 200 & 62061 & 55855 & 61440 & 606 & 324 & 930 & 60510 \\
\hline \multirow[t]{4}{*}{20} & 0 & 49300 & 44370 & 48807 & 606 & 300 & 906 & 47901 \\
\hline & 50 & 74733 & 67260 & 73986 & 606 & 381 & 987 & 72999 \\
\hline & 100 & 72283 & 65055 & 71560 & 606 & 462 & 1068 & 70492 \\
\hline & 200 & 69017 & 62115 & 68327 & 606 & 624 & 1230 & 67097 \\
\hline
\end{tabular}

Nota: Precio del zanahoria al momento de la cosecha fue de US $\$ 1.1 / \mathrm{kg}$, Costo del Nitrógeno por Kilogramo fue de US $\$ 1.62$ (Costo de la urea fue de US $\$ 37.3 / 50 \mathrm{~kg}$ ), para costo de lombricomposto, US $\$ 0.015 \mathrm{Gs} / \mathrm{kg}$ y el costo fijo fue de US\$606/ha.

\section{CONCLUSIÓN}

De los resultados de análisis de varianza, hubo diferencia significativa al $1 \%$ para $\mathrm{N}$ y al $5 \%$ para M.O. en el rendimiento del tubérculo. En el tratamiento sin M.O., se observó alto rendimiento con $100 \mathrm{kgN} / \mathrm{ha}$, mientras que fue de $50 \mathrm{kgN} /$ ha para el tratamiento con M.O.

Sobre análisis de tejido vegetal, de los resultados de análisis de varianza, hubo diferencia significativa al $1 \%$ no sólo para $\mathrm{N}$ sino también para M.O. en la absorción del N, $\mathrm{P}$ y Mg en el tubérculo. Pero, se considera que hubo efecto diluido para la absorción del P en el tubérculo, teniendo en cuenta alto rendimiento observado en los tratamientos con M.O. Por otra parte, la absorción del Ca y $\mathrm{Mg}$ en el tubérculo en los tratamientos con M.O. fue más alta que la absorción en los sin M.O., teniendo en cuenta los elementos liberados o aplicados por 20t/ha del lombiricomposto.

Sobre la evaluación de la aplicación de los dos materiales, fue el más bajo para el beneficio neto en el testigo sin aplicación del $\mathrm{N}$ ni lombricomposto. Se observó alto beneficio neto en el tratamiento con $100 \mathrm{kgN} /$ ha sin lombicomposto, pero el más alto el beneficio con $50 \mathrm{kgN} /$ ha asociado con lombricomposto.

\section{Agradecimiento}

Al realizar el experimento, Prof. Ing. Agr. Juan Alberto Bottino Fernández (Director Académico de la Facultad de Ciencias Agropecuarias y Desarrollo Rural, Universidad Nacional de Pilar) nos ayudó mucho. Le agradecemos a él.

\section{REFERENCIAS}

Álvaro Cordero. V. 1993. Fertilización y Nutrición mineral del arroz. Universidad de Costa Rica. San José. Costa Rica. pp. 35-42

Arias, Jiménez. C. A. 2007. Suelos Tropicales. Editorial Universidad Estatal a Distancia. San José. Costa Rica. 166. p

CENSO AGROPECUARIO NACIONAL (2008) VOLUMEN I SAN LORENZO - PARAGUAY AÑO 2009

http://www.mag.gov.py/Censo/Book\%201. pdf. $105 \mathrm{p}$

Huerto Familiar-Cultivo de Cebolla, Por Félix Maocho, Descripción general http://felixmaocho.wordpress.com/2009/0 6/02/huerto-familiar-cultivo-de-lacebolla/

La Granja. Revista Técnica y de Negocios de la Producción Granjera en Paraguay. $13 \mathrm{p}$ Proyecto DERMASUR (Desarrollo Rural y Mejoramiento Ambiental del Sur de 
Pilar). 1999. Guía Técnica sobre Cebolla para la Zona del Ñeembucu. Pilar Ñeembucú. Ministerio de Agricultura y Ganadería y Agencia Cooperación Internacional del Japón (JICA). 14 p

Proyecto DERMASUR (Desarrollo Rural y Mejoramiento Ambiental del Sur de Pilar). 1999. Estudio de Suelo" características Físico
- Químicas de los suelos del Área del Proyecto DERMASUR". Pilar - Ñeembucú. Ministerio de Agricultura y Ganadería y Agencia Cooperación Internacional del Japón (JICA). $39 \mathrm{p}$ 